\author{
César Luis Díez Plaza ${ }^{1}$ \\ Instituto Cervantes, Belgrado \\ Serbia
}

\title{
LECTORADOS DE ESPAÑOL EN SERBIA Y MONTENEGRO (1991-2019)
}

\begin{abstract}
Resumen
El objetivo de este trabajo es trazar una historia de los lectorados de español en Serbia y Montenegro durante el período 1991-2019, ubicados en cuatro universidades diferentes: Belgrado, Kragujevac, Novi Sad y Nikšić. También se analiza la relación entre el término español lector y el serbio lektor y se postula la hipótesis del (re)establecimiento de los lectorados como una acción exitosa de diplomacia cultural (académica).
\end{abstract}

Palabras clave: Serbia, lector, lectorado, español, diplomacia cultural.

\section{Objetivos y límites de este trabajo}

El objetivo de este trabajo es presentar el panorama de los lectorados de español en las universidades de Belgrado, Kragujevac, Novi Sad y Nikšić durante un período de casi treinta años: desde el curso académico 1991/92 hasta el curso académico presente 2019/20.

\footnotetext{
${ }^{1}$ cdplaza@cervantes.es
} 
Los límites geográficos señalados excluyen del estudio los lectorados situados en otros territorios de la antigua Yugoslavia (Croacia, Bosnia y Herzegovina, Eslovenia y Macedonia). En concreto, se analizarán los casos de lectorados existentes en la República Federal de Yugoslavia (1992-2003), en la Unión Estatal de Serbia y Montenegro (2003-2006) y en las actuales República de Serbia y República de Montenegro.

De la misma manera, los límites cronológicos marcados (19912019) también dejan fuera un posible estudio de los lectorados que existieron en las universidades de la República Socialista Federativa de Yugoslavia (1945-1992) durante gran parte de su existencia (a excepción del curso 91/92 para el caso belgradense) y algunos otros precedentes que pudieran existir. Por ejemplo, Delgado Gómez-Escalonilla nos menciona: «[E]ntre las escasas novedades que se registraron en el curso de aquél [1934] año estaban: la creación de lectorados en Osaka (Japón), Oxford (Gran Bretaña) y Sarajevo (Yugoslavia) [...]» (1991 [2002]: 88).

En una investigación más amplia sobre los lectorados en la RSFY, por ejemplo, se enmarcaría la larga historia de lectores y profesores de países de habla hispana en la Universidad de Belgrado que empezaría en 1951 cuando se le encargaron las clases al profesor español José Bort Vela. Posteriormente, durante el curso académico 1962/63 el español recibiría el estatus de segunda lengua extranjera dentro del programa de la Cátedra de Romanística. La responsable de estos estudios era la profesora Ljiljana Pavlović Samurović y las clases de «prácticas» (la ocupación de un lector) las impartiría Juan Octavio Prenz, profesor argentino que ejerció en los períodos de 1962-1967 y 1975-1980). En 1971, se crearía el Grupo de Lengua y Literatura Española como una licenciatura de cuatro años de estudios dentro del Departamento de Lenguas Romances (Soldatić \& Donić 2011: 31; Bošković 2017b: 395). En dicha estructura impartirían clases el ya citado Juan Octavio Prenz y Silvia Todorović, profesora peruana que ejerció desde el curso 1971/72 hasta el curso 2009/10.

Además de establecer una cronología de los diferentes lectorados y los programas, estatales o universitarios que permitieron establecerlos, también se presentará — con intención de proporcionar un marco general en el que situar la cronología mencionada- una interpretación sobre la relación existente entre el término español lector y el serbio lektor.

\section{Un inicio muy personal}

En los primeros días de octubre de 1995, tras una charla en el bar de la facultad con los compañeros de promoción sobre un posible futuro 
profesional al acabar los estudios, un estudiante de quinto y último año de Filología Hispánica de la Universidad Autónoma de Madrid (UAM) decidió estudiar una lengua poco conocida con la vaga idea de que esa le pudiera ayudar a conseguir un puesto en algún entorno académico.

Con dicho propósito en mente, se dirigió al edificio del rectorado de la universidad que también albergaba en aquel momento las dependencias del Servicio de Idiomas de la UAM para ver las lenguas ofertadas ${ }^{2}$. En una de las puertas de aquel departamento, se encontró con un nombre bastante largo, «serbocroata» (srpskohrvatski), que le llamó la atención. Además, tuvo la suerte de que aquella puerta estuviera abierta y que dentro se encontrase la profesora de aquella lengua, Dragana Bajić, quien amablemente le explicó el significado de aquel nombre. Por aquel entonces, aquel estudiante sólo tenía una idea muy vaga (formada con las noticias de los medios de comunicación) sobre lo que significaba Yugoslavia y lo que en ese momento estaba ocurriendo en los territorios que había formado la RFSY (guerras de los Balcanes). Aún así, el estudiante muy motivado por la charla con la profesora decidió matricularse inmediatamente en esa lengua, empezando un aprendizaje de la misma que dura hasta el presente.

En la Universidad Autónoma de Madrid (UAM), la historia del lectorado de serbocroata arranca a principios de los años 80 y en él estuvieron como lectoras las profesoras Jelena Rajić (1981/82), Marina Ljujić (1987/88-1988/89), Aleksandra Mančić (1989/90-1990/91) y Dragana Bajić (1991/92-1997/98). Una descripción muy completa de los estudios de serbística en la España actual y su relación con los de hispanística en Serbia se puede encontrar en un reciente artículo de Aleksandra Mančić (2016).

Al finalizar el año académico (y con él su carrera universitaria), y gracias a las gestiones realizadas por su profesora, el estudiante tuvo la oportunidad de viajar a Belgrado para asistir como estudiante a la XXV edición del Skup Slavista en la Universidad de Belgrado. Dicha reunión científica tiene una larga tradición (en 2019 se ha celebrado la 49 edición) y, además de conferencias y ponencias científicas, ofrece durante tres semanas clases de lengua serbia para estudiantes de todo el mundo ${ }^{3}$.

\footnotetext{
${ }^{2}$ En la actualidad en el Servicio de Idiomas de la UAM se ofertan (según su página web) clases de alemán, chino, ELE, francés, inglés, italiano y portugués. En el curso en el que se inscribió el estudiante (1994/95) se ofertaban también otras lenguas, como árabe, polaco, ruso o serbocroata.
}

${ }^{3}$ Las informaciones sobre el Skup se pueden consultar en la página: http://slavistickodrustvo.org.rs 
En aquella edición de septiembre de 1995, a la que se incorporó nuestro estudiante, asistieron otros dos estudiantes de España y de casi una veintena de países diferentes, países como Corea del Sur, China, Francia, Italia, Japón, Gran Bretaña, Grecia, Rumanía, Rusia, Ucrania, etc. Es importante remarcar el carácter multinacional de una reunión que tenía lugar en un momento en que la RFY se encontraba todavía bajo sanciones económicas debido al conflicto en Bosnia y Herzegovina (que finalizaría el 15 de diciembre del mismo año). Algo que se podría considerar un éxito de la diplomacia pública y académica de las autoridades universitarias del país.

Durante aquellas tres semanas, el estudiante tuvo la oportunidad de conocer a la Jefa del Grupo de lengua española y literatura de la Facultad de Filología, Ljiljana Pavlović Samurović, y al Vicedecano de Relaciones Internacionales de dicha facultad y profesor de literatura, Dalibor Soldatić. Estos le explicaron la situación del español en aquel momento (con una única profesora que tuviera el español como lengua materna) y le ofrecieron incorporarse al departamento en calidad de lector extranjero. Aquella propuesta emocionó al estudiante que consiguió un ordenador para redactar e imprimir su currículum vitae y una carta de motivación destinada a la profesora Samurović, con la idea de incorporarse al puesto lo antes posible. Sin embargo, desde la Embajada de España en Belgrado se le comentó que, debido a las especiales circunstancias del momento, no parecía muy adecuado que un español trabajara como lector en la universidad.

Esta adversidad no desanimó al profesor Soldatić que, durante los siguientes tres meses, continuó con las gestiones encaminadas a conseguir la incorporación del estudiante. Por fin, la situación se solucionó y, a principios de enero de 1996, el profesor Soldatić envió un fax al futuro lector informándole de que se podría incorporar a la facultad el primero de febrero con las siguientes condiciones: «salario a nivel de profesor adjunto (docente); alojamiento en el Hogar de estudiantes; seguro médico; transporte local e inscripción gratuita en los cursos de lengua serbia y en los cursos de posgrado en la Facultad de Filología» ${ }^{4}$. Unas condiciones que le parecieron inmejorables a un recién licenciado.

Se puede conjeturar que una herramienta para conseguir el objetivo de la incorporación de este joven licenciado español fue aprovechar la similitud existente entre el término español lector y el serbio lektor. El

\footnotetext{
${ }^{4}$ Extraído del texto del fax recibido por el futuro lector.
} 
segundo, según la legislación serbia 5 , es la denominación que reciben los colaboradores que imparten clases en el marco de los programas de estudios de las lenguas extranjeras. Además, en dicha denominación, se identifican dos niveles: lector y lector superior. El primero se define en la acepción cuarta del vocablo lector del diccionario de la RAE: «4 m. f. En los departamentos universitarios de lengua modernas, profesor, generalmente extranjero que enseña y explica en su propia lengua». A esta ajustada definición del DRAE, en el contexto propiamente español, se podría añadir el concepto de que el lector representa una cierta «institucionalidad» ya que es un órgano administrativo español (un ministerio, una agencia ministerial o una universidad) quien envía al lector, colaborando en su mantenimiento a través de una ayuda económica.

Gracias a la documentación remitida por la Universidad, el ya casi lector no tuvo problemas para conseguir el visado en la Embajadayugoslava en Madrid, y desde su llegada, el personal de la Embajada española en Belgrado, en especial el agregado de prensa, José Miguel Palacios (que, junto con el profesor Soldatić, había contribuido a la resolución de la situación), le prestó su ayuda en todo momento. El alojamiento mencionado resultó ser una acogedora habitación en la residencia de estudiantes Rifat Burdžević 6 , en la que el lector pasaría los siguientes seis años. En la residencia también se encontraban otros lectores extranjeros de la facultad — profesores de chino, polaco, ucraniano, inglés o turco-.

Una triste anécdota puede ilustrar el compromiso de las instituciones locales, incluida la administración de la residencia, con aquel colectivo internacional. Durante el tiempo que duró el bombardeo de la OTAN de 1999 sobre Yugoslavia, el lector, al igual que otros muchos de sus compañeros, tuvo que abandonar el país, dejando en aquella habitación todas sus posesiones del momento (en especial, muchos libros de segunda mano). A su vuelta, casi cuatro meses después, todo lo que se había quedado en la habitación permanecía en su sitio, no faltaba nada. Era como si hubiera salido de aquella estancia la tarde de antes. Otro momento emocionante fue cuando el Decano de la Facultad reunió a todos los lectores extranjeros que habían vuelto al país después del bombardeo o que, incluso, se habían quedado allí durante el mismo para agradecerles su labor y su compromiso con la institución.

\footnotetext{
${ }^{5}$ En la ley serbia se puede leer: „Zvanja saradnika u izvođenju nastave u okviru studijskih programa stranih jezika su: lektor i viši lektor."

${ }^{6}$ La residencia lleva el nombre de un héroe partisano de la II Guerra Mundial y fue la primera en abrirse tras la IIGM. [https://sc.rs/sc/rifat-burdzevic/]
} 


\section{Lectorados del MAE(C) y la AECI(D) ${ }^{7}$ en la Facultad de Filología de Belgrado}

Bošković (2017) proporciona una descripción de la historia de los estudios de español en la Facultad de Filología de Belgrado

El año 1971 marcó el comienzo de los estudios de Filología Hispánica con la creación del Grupo de Lengua y Literatura Española con programa de licenciatura de cuatro años de estudios en la Facultad de Filología de Belgrado. Este formó parte del Departamento de Lenguas Romances hasta el año 2000, cuando se desarrolló en la Cátedra de Estudios Ibéricos, que hoy en día forman el Departamento de Lengua Española y Literaturas Hispánicas, el Lectorado de Lengua Portuguesa y el Lectorado de Lengua Catalana (Soldatić \& Donić 2011: 31).

La llegada del nuevo lector al Grupo de Lengua y Literatura Española de la Facultad de Filología (en enero de 1996) se podría considerar una reiniciación del trabajo de los lectores de español tras el paréntesis que marcaron las sanciones internacionales. Debido a estas, la ultima lectora de MAE que había estado en Belgrado (curso 1991/92), María Ángeles Alonso Zarza, fue trasladada a la universidad de Szeged en la vecina Hungría.

El nuevo lector ejerció su función con esta vinculación directa con la Universidad desde febrero de 1996 hasta noviembre de 1997, momento en que fue nombrado lector del Ministerio de Asuntos Exteriores de España (MAE) en la misma universidad. Puesto que ocuparía hasta septiembre del 2000, cuando el lectorado pasó del MAE a la Agencia Española de Cooperación Internacional para el Desarrollo (AECI).

Desde ese momento, en la Cátedra, ha habido una presencia casi ininterrumpida de lectores de español enviados por la AECI. En total, se trata de seis lectores, incluyendo en la lista al actual. Estos son: Hugo Marcos Blanco (2002/03-2004/05), Inmaculada Gallegos Polonio

\footnotetext{
${ }^{7}$ Se ha optado por poner la última sigla entre paréntesis ya que, durante el período estudiado en este trabajo (1996-2019) ambos organismos de la administración española cambiaron su denominación. En el caso de la Agencia Espańola de Cooperación Internacional para el Desarrollo: AECI, en el período 1988-2007 y AECID, desde 2007 al momento actual. En el caso del Ministerio de Asuntos Exteriores: MAE, en el período 1977-2004, MAEC (entre los ańos 2004 y 2018, la «C» corresponde a cooperación) y, desde 2018, se denomina Ministerio de Asuntos Exteriores, Unión Europea y Comunicación.

${ }^{8}$ En el período anterior (1951-1971), los estudios de español, así como los de las otras lenguas románicas, se encontraban dentro de la Facultad de Filosofía.
} 
(2005/06-2007/08), Montserrat Zamorano Llena (2008/09-2010/11), Luiza Valozic Nenadic (2011/12-2014/15), Federico Escudero Álvarez (2015/16-2017/18) y Francisco Capilla Martín (desde el curso 2018/19). El hecho de que, hasta el momento, la totalidad de estos lectores hayan consumido el período máximo de permanencia en el lectorado (tres años académicos) y que, en algunos casos, continúe la vinculación con la Universidad de Belgrado son datos objetivos que hablan de su satisfacción con el desarrollo de sus funciones dentro del lectorado.

$\mathrm{Al}$ analizar las fechas señaladas, hay un caso en el que parece que la lectora estuvo un curso más de los tres estipulados (2011/12-2014/15). Sin embargo, esa interpretación no sería exacta ya que en dicho período se produce el único hueco en la presencia en la Cátedra de lectores enviados por la AECI (o el MAE). Se trata del curso 2012/13. En ese año, como efecto de la crisis económica, se redujeron mucho las ayudas a los lectorados en todo el mundo.

Por lo que respecta al proceso de selección de lectores, el procedimiento se ha mantenido prácticamente invariable a través de los años. Tras la publicación en el BOE de las plazas de los lectorados vacantes y, en algunos casos, las de renovación, los candidatos pasan un proceso de selección en el que los responsables de las universidades receptoras darán el visto bueno a un candidato determinado incluido en una terna de aspirantes remitida a las autoridades académicas locales por la AECID. La Embajada actúa como representante de la Agencia en el país y es un punto de referencia para los lectores. El lector, tras su primer año, puede solicitar su renovación (hasta un máximo de tres años en total), procedimiento que realiza a través de la Embajada previo informe positivo de la Universidad.

Los informes redactados por los lectores para solicitar la renovación de la ayuda y las opiniones emitidas por los responsables de la Cátedra son una importante fuente documental para seguir la historia del lectorado y de la Cátedra, así como para conocer las actividades y proyectos en los que participan los lectores. Por ejemplo, en una carta remitida a la Embajada por el Jefe de la Cátedra en 2011, solicitando que se ampliara el número de lectores a dos, se puede leer como la Cátedra se había convertido en aquel momento en una de las más grandes de la región, con más de 600 estudiantes matriculados en los estudios de licenciatura en Lengua Española y Literatura Hispánicas y que otros 200 se encontraban estudiando español como segunda lengua. Para atender a todos estos estudiantes, la Cátedra contaba en aquel año, además de con los catedráticos y profesores titulares, con cinco lectores españoles (uno 
enviado por la AECI, otro por la Universidad de Granada y tres con un contrato denominado «libre») y una lectora cubana.

Igualmente, en la documentación mencionada, los lectores señalan que el interés de los estudiantes por la lengua española se extiende en diferentes direcciones: traducción e interpretación, estudios lingüísticos, literarios y conocimientos culturales. Campos que coinciden con las materias que componen el plan de estudios ofertado por la Cátedra. Además, a partir del período 2012/13, se menciona el interés de los estudiantes por participar en programas internacionales de intercambio (como el programa Erasmus), impulsados desde el Decanato de la Facultad de Filología.

\section{Otros lectorados en la Cátedra de Belgrado}

Además de esta línea de lectores enviados por la administración española - MAE(C), AECI(D) - existe otra línea que es la de lectores enviados por universidades españolas, en concreto la Universidad de Granada. En esta universidad andaluza, funciona desde hace veinticinco años una Sección Departamental de Filología Eslava, en la que se impartió durante unos años lengua serbia; por ejemplo, Ivana Vučina Simović fue lectora en el Grupo de Filología Eslava de la Facultad de Filosofía y Letras de la Universidad de Granada durante el curso académico 2005/06 ${ }^{9}$. Los contactos entre el profesor español Rafael Guzmán Tirado y el profesor Soldatić fueron los que permitieron el intercambio de lectores entre ambas Universidades por medio de unos convenios que estuvieron en vigencia casi veinte años (1997-2017).

La primera lectora enviada por la Universidad de Granada a Belgrado fue María del Mar Campos Fernández-Figares (curso 1997/98) y después le siguieron otros diez lectores: Alicia Jiménez Mantsiu (1997/98-1998/99), Jorge Martínez Jiménez (2001/02-2002/03) Salomé Monasterio Morales (2003/04-2005/06), Luis Antonio Monzó Jiménez (2004/05-2016/17), Lucía Gil Villa (2005/06-2006/07), Cristóbal Díaz Beltrán (2006/07-2009/10, 2014/15-2016/17), Ana María García Gutiérrez (2007/08-2014/15), Raquel Lozano Pleguezuelos (2009/10-2014/15), Estela Castelló Pina (2013/14-2016/17) y Sara Toro (2014/15-2016/17).

Además de estos lectores, el Grupo de Español, y posteriormente la Cátedra de Estudios Ibéricos, contó con otra serie de lectores que

\footnotetext{
${ }^{9}$ En el plan de estudios publicado en la página web de la universidad granadina aparecen dos asignaturas optativas de serbocroata (Serbocroata I y II).
} 
no habían llegado en virtud de la firma de convenios, como son Álida Ares Ares, Pilar Dolado, Luz Marina Pallarés López (las tres durante la primera mitad de la década de los 90), Jairo Dorado Cadilla (2007/08) o la profesora cubana Jenny Perdomo (desde el curso 2001/02 hasta la actualidad).

Aunque en este trabajo no sea posible abordar en profundidad el tema, hay que señalar que la Cátedra de Estudios Ibéricos de la Facultad de Filología de Belgrado incluye en su plan de estudios a las lenguas catalana y portuguesa y, dentro de la estructura de la universidad, ambas cuentan con un lectorado. Las primeras conversaciones para conseguir el lectorado de catalán fueron impulsadas por el profesor Soldatić y tuvieron lugar en el último trimestre de 2002. Dicho lectorado se formalizaría con la firma de un convenio con el Instituto Ramón Llull ${ }^{10}$ en 2005. Desde ese momento, el lectorado ha contado con los siguientes lectores ${ }^{11}$ : Pau Bori (2005/06-2016/17), Áxel González (2010/11-2012/13) y Lea Feliu Pujol (desde el curso 2017/18 hasta la actualidad).

De igual manera, dentro de la Cátedra de Estudios Ibéricos se incluye también un lectorado de portugués. El primer lector de esta lengua fue el profesor brasileño Carlos do Amaral Freire (1997), que llegó gracias a las gestiones realizadas por el profesor Soldatić con la Embajada de Brasil. Después desempeñarían el puesto de lector Pericles Cunha y Veljko Prijić (2006/07-2007/08).

A partir de 2005 se firmaría un convenio con el Instituto Camoes ${ }^{12}$, por el cual llegarían los siguientes ocho lectores: André Cunha (2005/062012/13), Joana Margarida da Câmara (2007/08-2010/11), Mariana Faria (2008/09-2010/11), André Carvalhosa (2010/11-2011/12), Magda Barbeita (2012/13-2016/17), Clara Riso (2013/14-2015/16), Sofia Marinho (2014/15-2016/17) y José Carlos Canoa (desde el curso 2019/20).

\footnotetext{
${ }^{10}$ El Instituto Ramon Llull es una institución dependiente de la Generalidad de Cataluńa, del Ayuntamiento de Barcelona y del Gobierno Balear con el objetivo de promover la proyección exterior de la lengua y la cultura catalanas que fue fundada el 5 de abril de 2002.

${ }^{11}$ El autor agradece a la secretaria de la Cátedra, Izabela Beljić, el envío del listado de lectores.

${ }^{12}$ El Instituto Camões es una institución fundada en 1992 por el gobierno portugués para la promoción de la lengua portuguesa y de su cultura por todo el mundo.
} 


\section{Lectorado en la ciudad de Novi Sad}

Novi Sad es la segunda ciudad del país y capital de la provincia autónoma de Voivodina. La lengua espańola se estudia en la Facultad de Filosofía de dicha ciudad dentro del Departamento de Romanística. Este departamento tiene su origen en la Cátedra de lengua y literatura francesa que fue fundada en 1961 y que, en la actualidad, imparte asignaturas de espańol e italiano ${ }^{13}$ además de francés. La lengua rumana, aunque románica, posee su propio departamento ${ }^{14}$ debido a su estatus de lengua regional en Voivodina.

Teniendo en cuenta este contexto, en el que el espańol se había empezado a impartir en 1997, en 1998 desde la Embajada espańola en Belgrado, se empieza a tratar con la AECI la posibilidad de tener un lector espańol. En aquel momento era embajador Joaquín Pérez Gómez (que desempeńó el cargo entre junio de 1996 y diciembre de 2000).

La AECI aprobó la apertura del lectorado para el curso académico 1998/99 y, tras la convocatoria pública, el puesto de lector lo ocupó María del Mar Campos Fernández-Figares; quien, con anterioridad (durante el curso 1997/1998), había sido la primera lectora enviada por la Universidad de Granada a la Facultad Filología de Belgrado gracias al intercambio firmado entre estas universidades. En marzo de 1999, debido a los bombardeos de la OTAN, la lectora tuvo que abandonar la ciudad, reincorporándose posteriormente para finalizar el curso académico. Debido a su condición de doctor, la lectora impartió —además de las clases de lengua - clases de literatura en distintos niveles y dictó distintas conferencias (en Belgrado y Budva) sobre Federico García Lorca, de quien se cumplía el aniversario de su nacimiento ${ }^{15}$.

Tras la marcha de esta lectora, hubo un paréntesis en el lectorado durante los cursos 1999/00 y 2000/01. A principios de 2001, se retomó el proyecto del lectorado y en abril de ese ańo se firmaba un Memorándum de Entendimiento entre la Universidad de Novi Sad y la AECI por el que se constituía el lectorado dentro del Departamento de Romanística. Dicho memorándum fue firmado por la Rectora de la Universidad, Fuada Stanković (rectora entre 2001 y 2004), y el Embajador de Espańa en Belgrado, Mariano García Muńoz (embajador entre 2000 y 2004).

\footnotetext{
13 http://www.ff.uns.ac.rs/sr/fakultet/odseci/romanistika/o-odseku. Último acceso noviembre 2019.

14 http://www.ff.uns.ac.rs/sr/fakultet/odseci/rumunistika. Último acceso noviembre 2019.

${ }^{15}$ Comunicación personal de María del Mar Campos al autor de este trabajo.
} 
Tras haberse aprobado la reanudación de la actividad del lectorado de la AECI para el curso 2001/2002 y convocarse el concurso público, se nombró como lectora a Loreto García Pardo (2001/02-2003/04). Después desempeńarían esta misma labor tres lectores más: Román García Pecharromán (2003/04-2006/07), Natalia Sánchez Radulović (2007/082009/10) y María José Nadal López (cursos 2010/11 y 2011/12).

En febrero de 2012, la AECI comunicó a la Embajada espańola en Belgrado que, debido a la necesidad de efectuar recortes en el programa de lectorados para la convocatoria de 2012/13, se debería amortizar uno de los lectorados de los tres existentes en el país (Belgrado, Kragujevac y Novi Sad). Debido a una serie de circunstancias, fue el lectorado de Novi Sad el que se suprimió.

Sin embargo, dicho suceso no significó que decayera el interés por el estudio del espańol, ni que dejaran de matricularse alumnos en estos estudios, tanto en la modalidad de «segunda lengua», como en la de «asignatura optativa / lengua extranjera». Según los datos de la profesora Ivana Georgijev ${ }^{16}$, en los cinco últimos ańos académicos (desde el curso académico 2014/15 hasta el actual) se han matriculado en espańol como segunda lengua 216 alumnos, mientras que 688 lo han hecho como asignatura optativa.

Actualmente, para impartir clases de espańol, la Facultad de Filosofía de Novi Sad cuenta con dos lectores extranjeros: la boliviana Victoria Riveros Mitrović desde el ańo (2017/18) y el espańol Moisés Moreno Fernández (desde el ańo 2013/14).

\section{Lectorado en la ciudad de Kragujevac}

Kragujevac es la cuarta ciudad de Serbia (después de Belgrado, Novi Sad y Niš). El español empezó a estudiarse en la Universidad de Kragujevac en el año 2000 dentro de una estructura que formaba parte de la Universidad de Belgrado. A partir de 2002 se convirtió en un departamento independiente de la Facultad de Filología y Artes de la Universidad de Kragujevac. Entre los años académicos 2004/05 y 2006/07, el profesor argentino Fabián Vique impartió clases de español, como lector local, en dicho departamento.

\footnotetext{
${ }_{16}$ Datos obtenidos de la ponencia de la profesora Ivana Georgijev en la conferencia „Романске студије - јуче, данас, сутра“ / LES ÉTUDES ROMANES AUTREFOIS ET AUJOURD'HUI / ESTUDIOS ROMÁNICOS: ANTES Y AHORA / GLI STUDI DI ROMANISTICA TRA PASSATO E PRESENTE, organizada en la Facultad de Filosofía de Novi Sad los días 11 y 12 de mayo de 2019.
} 
En febrero de 2006, la Jefa del Departamento de Hispanística de la Facultad de Filología y Artes, Anđelka Pejović, escribió una carta a la atención de la Embajada de España y de la AECI solicitando la apertura de un lectorado de la AECI en dicho Departamento, que - en ese momentocontaba con unos 130 estudiantes de español como primera lengua y 150 como segunda lengua.

En 2007 se firmó un Memorando de entendimiento entre el embajador de España, José Riera Siquier, en representación de la AECI, y el rector de la universidad, Miloš Đuran y el decano de la Facultad de Filología y Artes por parte de la Universidad de Kragujevac. La apertura del lectorado de la AECI se efectuó a principios del curso 2007/08 y este fue ocupado, en primer lugar, por María José Nadal López (2007/082009/10) y después por Natalia Sánchez Radulović (2010/11-2011/12).

El lectorado, además de sus labores en la difusión del español, sirvió como un dinamizador de la actividad cultural relacionada con España, apoyado por la Embajada en Belgrado (en 2011, por ejemplo, se celebraron actividades culturales como «El día de la Mujer», «El día E» o «Vamos a Entremesear»).

En 2013, debido a la crisis económica, la AECI debió cancelar un gran número de lectorados, entre los que se encontraba el de Kragujevac. Para superar el obstáculo que podía suponer la falta de profesorado nativo se firmó, a iniciativa de la Vicedecana de relaciones y desarrollo de la Facultad y directora del departamento de español, Anđelka Pejović, un Convenio Marco entre la Facultad de Filología de la Universidad de Kragujevac y la Universidad de Jaén, por el que fue posible que la Universidad de Kragujevac contratara a lectores provenientes de la universidad andaluza.

En la actualidad, dicho acuerdo sigue vigente y, desde el momento de su entrada en vigor, han impartido clase en Kragujevac cinco lectores provenientes de esta universidad andaluza: Isabel López López (2013/14-2014/15), Ana María Arias (2015/16), Rodrigo Fernández Migalla (2016/17-2017/18), Teresa J. Ángeles Galiano (2018/19) y Nieves Moreno Rodado (desde el inicio del presente curso académico, 2019/20).

\section{Lectorado en la ciudad de Nikšić}

Nikšić es la segunda ciudad de la República de Montenegro (tras la capital del país). En 2010 se empezaron a dar los primeros pasos para conseguir abrir un lectorado en la Facultad de Filosofía de la Universidad 
de Montenegro (Filozofski Fakultet, Univerzitet Crne Gore), situada en Nikšić, donde ya se impartían clases de español como asignatura optativa desde el curso académico 2003/04. Como se describe en la obra del Instituto Cervantes Anuario del español en el mundo 2006-2007:

En Montenegro se imparten dos años de español como asignatura optativa en la Facultad de Filosofía de la Universidad de Nikšić. No existe un departamento de español como tal, a pesar de la demanda de español como segunda lengua. El número de estudiantes ronda los 230 (Menéndez Puente 2006: 288).

Según los responsables de la universidad, dicho programa suponía unos estudios bienales equivalentes a un nivel B2. A partir de ahí, la idea inicial era que el español superara ese estudio de dos años y se convirtiera en una segunda lengua extranjera dentro del marco de los estudios de italiano, francés o inglés con tres años de docencia, en los que se incluirían, además de la lengua, los estudios de civilización y cultura. Los argumentos para apoyar dicha idea eran que existía un creciente interés por el estudio de la lengua española y que durante el curso académico 2009/10 había habido 167 estudiantes matriculados en español. Un número que aumentaría hasta 600 teniendo en cuenta el momento de partida, curso académico 2003/04. Mientras que el objetivo a más largo plazo era la formación de una Cátedra de Español que pudiera evolucionar a una Cátedra de Estudios Ibéricos (siguiendo el ejemplo de Belgrado).

Para conseguir la institución del lectorado se firmó un Memorando de Entendimiento para la concesión de subvenciones y ayudas a Lectorados de español MAEC-AECID entre la AECI y la Facultad de Filosofía de la Universidad de Montenegro. Como es norma general en este tipo de documentos el anexo al memorando incluía el perfil académico y profesional del lector (orientado a que pudiera ocuparse también de materias relacionadas con la civilización y la cultura), la descripción del departamento de español, las materias que debería impartir el lector, el tipo de contrato, el calendario académico (comienzo y finalización del curso y períodos de vacaciones) y un apartado de observaciones. En representación de la AECID, firmó el memorándum el Embajador de España en Serbia, Ignacio de Palacio España (embajador entre 2008 y 2011).

Por parte de la AECID, en abril de 2010, se autorizó la apertura del lectorado para el año académico siguiente 2011 /12 y el puesto aparecería 
en la convocatoria del BOE de 24 de febrero de 2012. La persona que ocupó el puesto de lector fue Gemma Querol Calvet, que renovaría para el curso 2012/13. Dicha profesora se encargó, a partir del 1 de septiembre de 2011, de impartir clases a tres grupos con diferentes niveles según del Marco Común Europeo de Referencia (MCER), desde al A1 al B2. Las sesiones de los cursos tuvieron una duración de dos horas y media dos veces a la semana. Otra de las actividades que asumió la lectora fue la coordinación y difusión de los exámenes DELE en colaboración con el IC de Belgrado.

En la actualidad, los estudios de español en la Universidad de Nikšić se encuadran en la Facultad de Filología ${ }^{17}$, fundada en $2015^{18}$. A la misma se adjuntó el Instituto para lenguas extranjeras de Podgorica ${ }^{19}$.

El caso de Nikšić se ha tratado en este trabajo, aunque no se encuentre en el territorio descrito en los objetivos marcados para este trabajo, ya que la iniciativa para poner en marcha el proyecto surgió en el entorno de la Facultad de Filología de Belgrado y el profesor Soldatić, y los trámites para conseguirlo los gestionó la Embajada espańola en Belgrado que también se ocupa de la representación diplomática en Montenegro. Una descripción de la situación de los estudios de espańol en Montenegro se puede encontrar en el artículo de Ivana Bošković (2017a).

\section{A modo de conclusión}

El objetivo final de las páginas anteriores no es establecer una mera cronología de nombres a través de casi treinta años de historia, sino constatar el éxito de un proyecto: la consolidación de los estudios de español en la enseñanza superior en Serbia. Un proyecto en el que los lectores han jugado un gran papel, desarrollando con entusiasmo una importante labor académica y motivando a varias generaciones de estudiantes.

Y, para que los lectores hayan podido desarrollar dicha labor, fue necesario establecer los programas de lectorados con el fin de conectar a las dos administraciones - la española y la serbia- a través de los canales abiertos por la Embajada española en Belgrado y las universidades locales. Se trata, sin lugar a dudas, de un importante ejemplo de diplomacia cultural en el que una de las partes detecta una necesidad y la cubre recurriendo

\footnotetext{
${ }^{17}$ Dirección electrónica de la facultad: https://www.ucg.ac.me/objava/org/28/poz/info

${ }^{18}$ Službeni list CG br. 49/15 od 20. 08. 2015.

${ }^{19}$ http://www.obrazovanjeodraslih.me/index.php
} 
al apoyo de la otra parte. En este caso, los responsables universitarios serbios se dieron cuenta de lo importante que era contar con profesores nativos que colaboraran en el proceso de aprendizaje de los cada vez más numerosos estudiantes y les despertaran el interés por la realidad cultural y social española y se pusieron en contacto con las autoridades españolas para conseguir crear las estructuras que permitieran la presencia de estos profesores.

Pero, incluso antes de que los programas se establecieran, se puede hablar de gran ejemplo de diplomacia cultural (o pública si se prefiere), ya que se consigue que, en un momento en el que Serbia permanecía aislada de la comunidad internacional debido a las sanciones (mediados de los años 90 del siglo pasado), se abriera una pequeña ventana en el mundo académico gracias a la reactivación de los lectorados.

De esta manera, en el momento descrito al principio de este artículo (1996), cuando el país estaba bajo sanciones por los acontecimientos políticos y bélicos, la Facultad de Filología de Belgrado consiguió cubrir ese puesto reconocido en su legislación, el de lektor, con un español que, en aquellos momentos, no se podría considerar lector, ya que no había sido enviado por ninguna rama de la administración española. El mérito de este éxito de diplomacia cultural o académica fue del profesor Dalibor Soldatić, en su doble faceta de Vicedecano de Relaciones Internacionales de la Facultad y de profesor del Grupo de Español, quien consiguió que ese español con el que se comenzaba el artículo (y que no es otro que el que firma estas líneas) llegara a Belgrado. Tras aquel primer éxito inicial, también fue el profesor Soldatić el que, con el decidido apoyo de la Embajada española, consiguió que el lectorado de la Facultad se institucionalizara primero a través del MAE y después de la AECI. Y, consolidada la situación de Belgrado, impulsó muy activamente la apertura de los otros tres lectorados: Novi Sad, Kragujevac y Nikšić. No sería posible escribir la historia de la enseñanza del español en este entorno sin hacer referencia a toda su labor y dedicación.

\section{Agradecimientos}

La redacción de este trabajo no hubiera sido posible sin la ayuda prestada por muchas personas pertenecientes a los diferentes ámbitos en los que se desarrolla esta historia. Me gustaría agradecer toda su ayuda a Carmen Álvarez Rodríguez, Vesna Mastalo y Marija Minić de la Embajada de España en Belgrado; a Izabela Beljić, Jelena Filipović, Anđelka Pejović y Jelena Rajić de la Cátedra de Estudios Ibéricos de la 
Facultad de Filología de Belgrado, y a Mercedes de la Chica y Sebastián J. Bruque de la Universidad de Jaén. Igualmente quiero agradecer a los antiguos lectores todas las informaciones que, amablemente, me han proporcionado. Muchas gracias a María Ángeles Alonso, Teresa J. Ángeles, María del Mar Campos, Jairo Dorado Cadilla, Federico Escudero, Loreto García, Hugo Marcos, Salomé Monasterio, Luis A. Monzó, Román Navarro, Sara Toro y Luiza Valožić Nenadić. También quiero agradecer su ayuda y apoyo a Dragana Bajić, a Aleksandra Mančić, a Guillermo Menéndez (del IC de Belgrado) y a José Miguel Palacios, de quien partió la idea de escribir estas líneas. Por supuesto, también quiero agradecer al profesor Soldatić su ayuda para escribir este artículo y, sobre todo, que hace veintitrés ańos me diera la oportunidad de llegar a Belgrado.

Finalmente, quiero pedir disculpas por si he omitido algún nombre en las relaciones o he equivocado alguna fecha. Asumo plenamente la responsabilidad de esas posibles omisiones o errores.

\section{REFERENCIAS BIBLIOGRÁFICAS}

Bošković 2017a: Ivana Bošković. «Enseñar español en Montenegro. El español entre las montañas y las playas montenegrinas». María del Carmen Méndez Santos \& María del Mar Galindo Merino (Eds.). Atlas del ELE. Geolingüística de la enseñanza del español en el mundo. (Volumen I. Europa oriental). Madrid: EnClaveELE, 1-6.

Bošković 2017b: Ivana Bošković. «Enseñar español en Serbia. El español en la encrucijada balcánica». María del Carmen Méndez Santos \& María del Mar Galindo Merino (Eds.). Atlas del ELE. Geolingüística de la enseñanza del español en el mundo. (Volumen I. Europa oriental). Madrid: EnClaveELE, 395-410.

Delgado Gómez-Escalonilla 2002: Lorenzo Delgado Gómez-Escalonilla. Acción cultural y política exterior. La configuración de la diplomacia cultural durante el régimen franquista (1936-1945). Tesis de doctorado. Madrid: Universidad Complutense de Madrid (CD-ROM: 1992).

Mančić 2016: Aleksandra Mančić. «Los estudios de serbística en España en el siglo XXI y los estudios de hispanística en Serbia: traducción de ideas y transculturalismo académico. Apuntes iniciales». Anđelka Pejović et al. (Eds.). Estudios hispánicos en la cultura y ciencia serbia - Actas de la primera conferencia nacional de hispanistas serbios (Facultad de Filología y Artes de Kragujevac, 28-29 noviembre 
2014). Kragujevac: Facultad de Filología y Artes de Kragujevac, 441-462.

Méndez Santos 2017: María del Carmen Méndez Santos \& María del Mar Galindo Merino (Eds.). Atlas del ELE. Geolingüística de la enseñanza del español en el mundo. Volumen I. Europa oriental. Madrid: EnClaveELE.

Menéndez Puente 2007: Guillermo Menéndez Puente. «El español en Serbia y Montenegro, Bosnia-Herzegovina y Macedonia». Instituto Cervantes (Ed.). Anuario del español en el mundo 2006-2007. Madrid: Instituto Cervantes / Barcelona: Plaza \& Janés, Círculo de Lectores, 287-294.

Soldatić \& Donić 2011: Dalibor Soldatić \& Željko Donić. Svet hispanistike: uvod u studije, Beograd: Zavod za udžbenike.

\section{SPANISH LECTORATES IN SERBIA AND MONTENEGRO}

(1991-2019)

\section{Summary}

The objective of this work is to trace a history of the Spanish lecturers in Serbia and Montenegro during the period 1991-2019, located in four different universities: Belgrade, Kragujevac, Novi Sad and Nikšić. The relationship between the Spanish term lector and the Serbian lektor is also analyzed and the hypothesis of (re) establishment of lectorates is postulated as a successful action of cultural (academic) diplomacy.

Keywords: Serbia, lector, Lectorado, Spanish, cultural diplomacy. 